\title{
Application of a temporary intestinal storage device in a small intestine gunshot wound dog model
}

\section{Weihang Wu}

Fuzhou General Hospital of Nanjing Military Command Area: 900th Hospital of PLA

\section{Mingwei Wang}

fujian medical university

\section{Weikang Zhou}

Fujian Medical University

\section{Yuewen Zhu}

Xiamen University Medical College

Tianyu Lin

fujian medical university

\section{Shiyao Zheng}

Fujian medical university

Nan Lin

Fuzhou General Hospital of Nanjing Military Command Area: 900th Hospital of PLA

\section{Weijin Yang}

Fuzhou General Hospital of Nanjing Military Command Area: 900th Hospital of PLA

\section{Chunhong Xiao}

Fuzhou General Hospital of Nanjing Military Command Area: 900th Hospital of PLA

\section{Yu Wang ( $\sim$ flyfishwang@hotmail.com )}

Department of General Surgery,Dongfang Hospital,Xiamen University,Fuzhou,Fujian China

\section{Research article}

Keywords: intestinal gunshot wound, temporary intestinal storage, intestinal tube reconstruction, first aid at the battlefield, early enteral nutrition

Posted Date: January 20th, 2021

DOl: https://doi.org/10.21203/rs.3.rs-149056/v1

License: (9) This work is licensed under a Creative Commons Attribution 4.0 International License. Read Full License 


\section{Abstract}

Background: We aimed to verify the feasibility of a novel temporary intestinal storage device (TISD) using a simple intestinal gunshot wound model.

Methods: Ten female beagle dogs were fasted for 12 hours and anesthetized. An incision protector was inserted into a $10-\mathrm{cm}$ abdominal incision. The small intestine was exposed to the body by natural drooping. An automatic rifle was used to shoot the intestine from a distance of 25 meters to introduce a simple intestinal gunshot wound. The three phases of first aid for war injuries were followed: Care Under Fire, Tactical Field Care, and Tactical Evacuation Care. For Tactical Field Care, a novel TISD was used to reconstruct the ruptured intestine, and necrotic intestinal tissue was stored. The abdominal cavity was temporarily closed, and the abdomen was opened for exploration 4 hours after surgery. Treatment time was observed during Care Under Fire, transfer time was observed from Tactical Field Care to Tactical Evacuation Care, rescue was observed during Tactical Evacuation Care, and the treatment time of each intestinal segment was measured. After 4 hours, intestinal vitality was observed, and the heart, liver, spleen, lung, kidney, stomach, normal intestine, and necrotic intestine were examined before and 4 hours after surgery by light microscopy. The broken ends of the intestine were connected to the intestinal reconstruction device before and 4 hours after surgery and were examined by transmission electron microscopy.

Results: The processing time of Care Under Fire was $41.55 \pm 10.46$ seconds, which is shorter than the maximum time limit of the battlefield first aid principle. Transit time from Care Under Fire to Tactical Field Care transit was $60.78 \pm 15.95$ seconds, which is shorter than the battlefield first aid principle. The treatment time of Tactical Field Care was $29.75 \pm 5.13$ minutes, and the reconstruction time of each intestinal segment was $4.44 \pm 0.31$ minutes. One dog died of anesthetic overdose, two died of splenic bleeding, and the rest completed all phases. The abdominal cavity was explored 4 hours after surgery, and the TISD was positioned. Intestinal tract reconstruction was normal, and no obvious necrosis was observed. Necrotic intestine had the same vitality as before storage. With light microscopy, the heart, liver, spleen, lung, kidney, and stomach showed no obvious necrosis, inflammatory cell infiltration, or necrosis of normal intestine before and after surgery. Before and 4 hours after surgery, intestinal necrosis involved local necrosis of villi and tissues, and marked inflammatory cell infiltration. Transmission electron microscopy showed that the villi of the intestinal stump connected to the TISD before surgery were intact, and no obvious necrosis was observed. The villi of the intestinal stump were moderately damaged after surgery, and focal necrosis was observed.

Conclusions: The novel TISD can be used in the emergency treatment of simple small intestine gunshot wounds in beagle dogs and can prevent further deterioration after intestinal injury. Background: We aimed to verify the feasibility of a novel temporary intestinal storage device (TISD) using a simple intestinal gunshot wound model.

\section{Full Text}


Due to technical limitations, full-text HTML conversion of this manuscript could not be completed. However, the manuscript can be downloaded and accessed as a PDF.

\section{Figures}

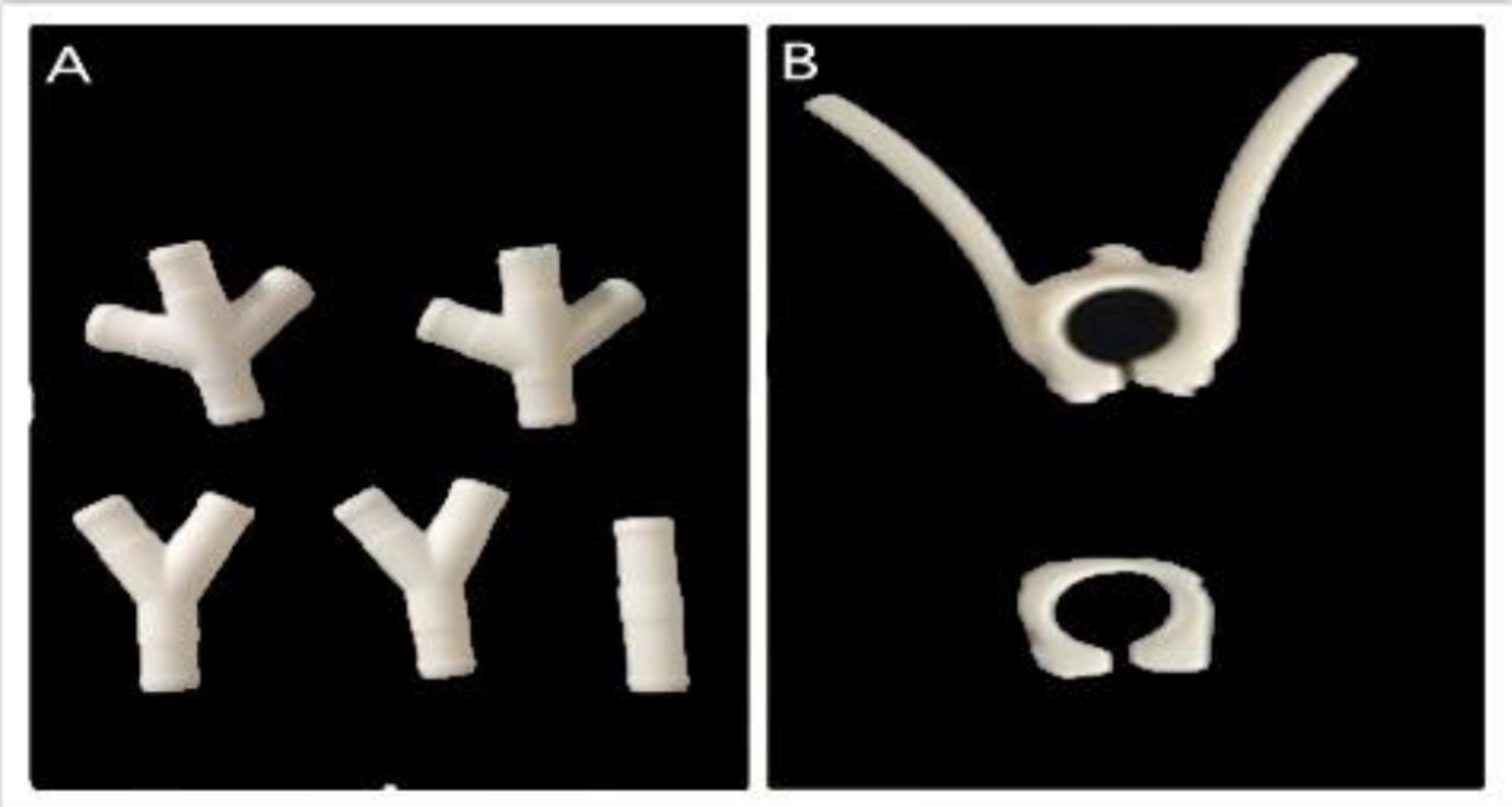

\section{Figure 1}

Novel temporary intestinal storage device. A. Intestinal tube storage tube. B. Storage tube fixing device. 




Figure 2

Establishment of a simple small intestine gunshot wound model. $20 \mathrm{~A}$. The intestine is exposed outside the body. B. Marking of the shooting point. C. Rifle shooting. 




\section{Figure 3}

Temporary abdominal closure with forceps after hemostasis. 




Figure 4

Temporary bowel reconstruction. A. Temporary intestinal reconstruction. B. Schematic of temporary intestinal reconstruction. 


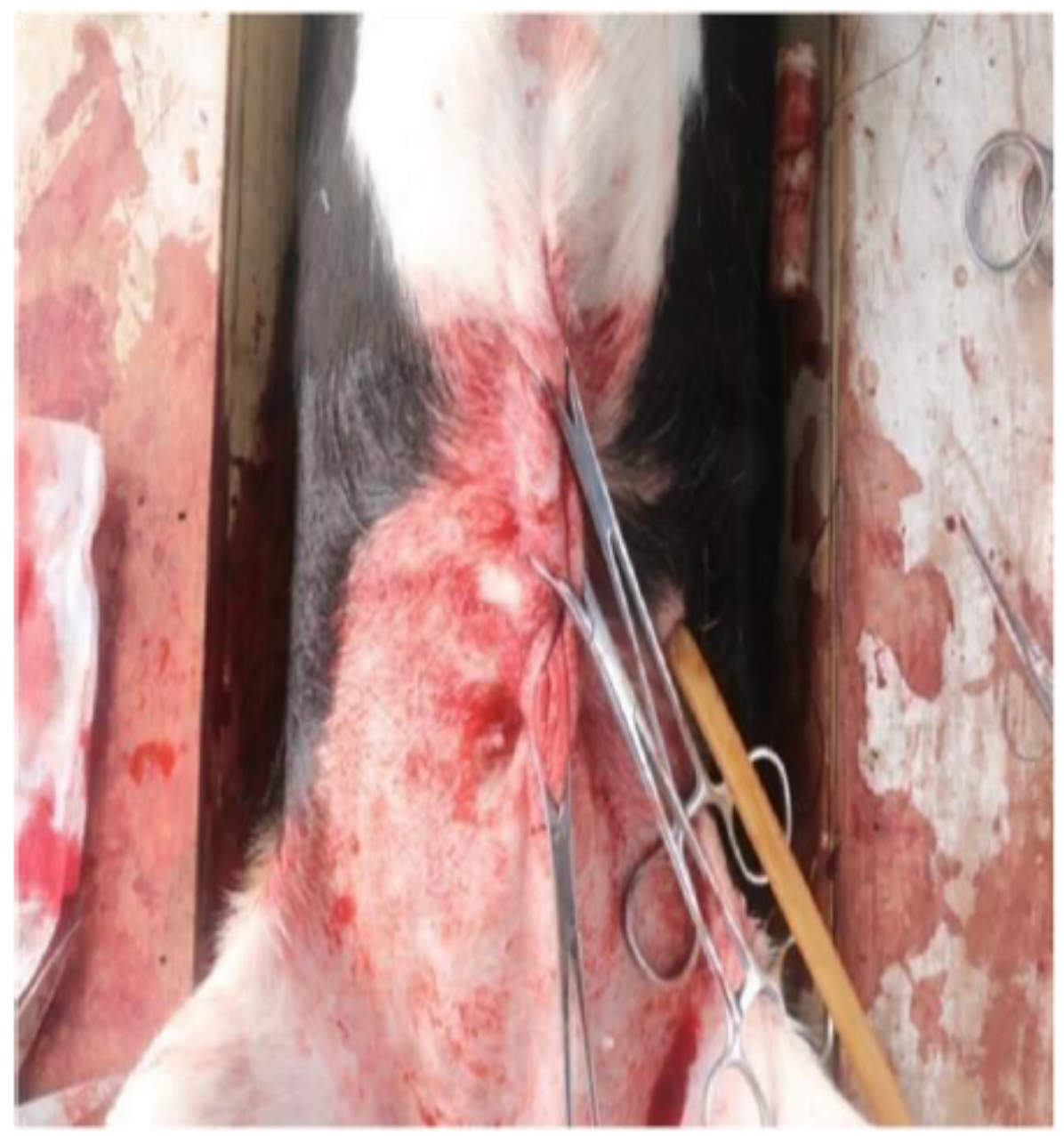

Figure 5

The towel clamp temporarily closes the abdominal cavity. 

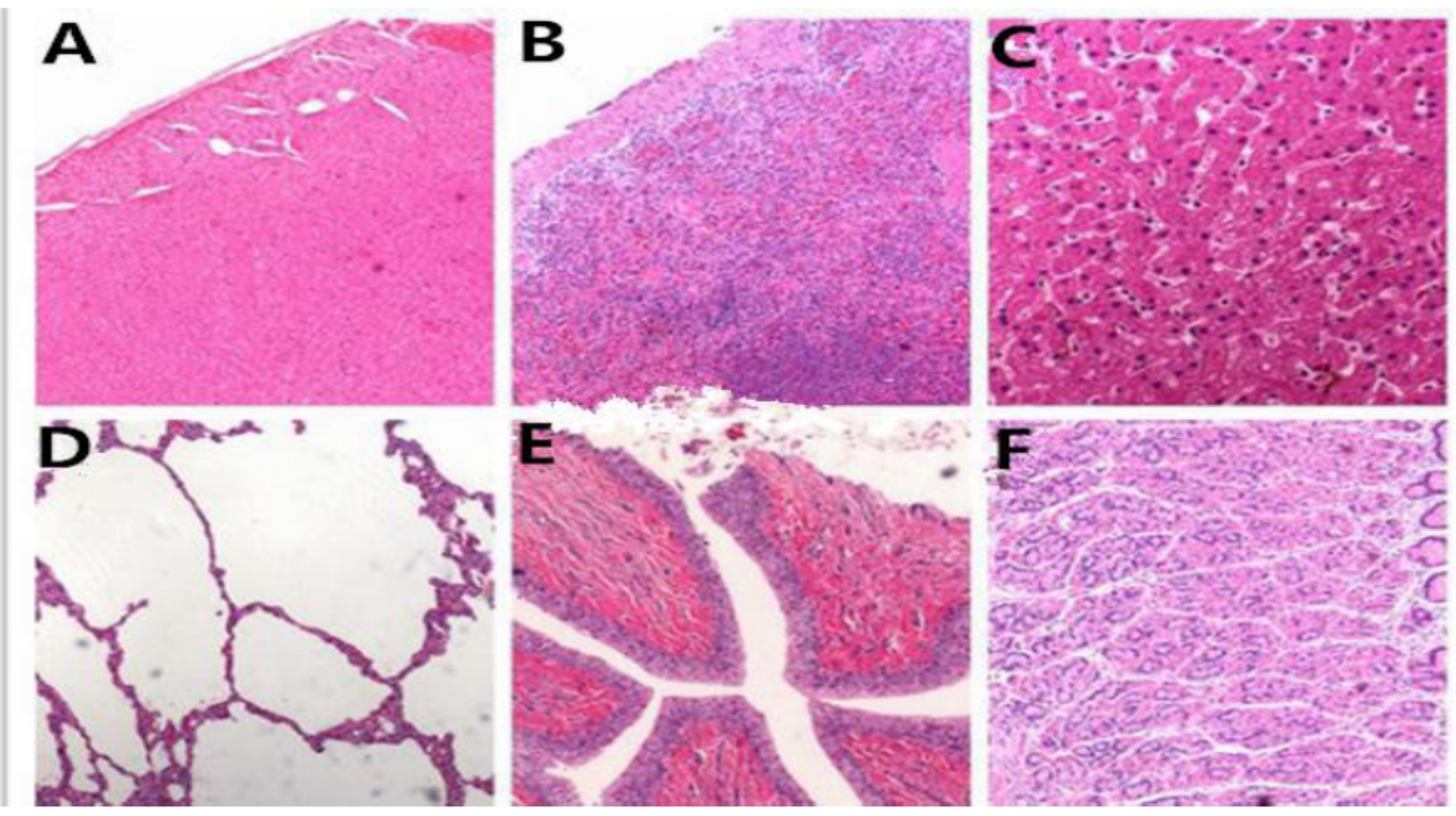

\section{Figure 6}

Pathology of multiple organs with light microscopy. A. Heart. B. Spleen. C. Liver. D. Lung, E. Kidney. F. Stomach.
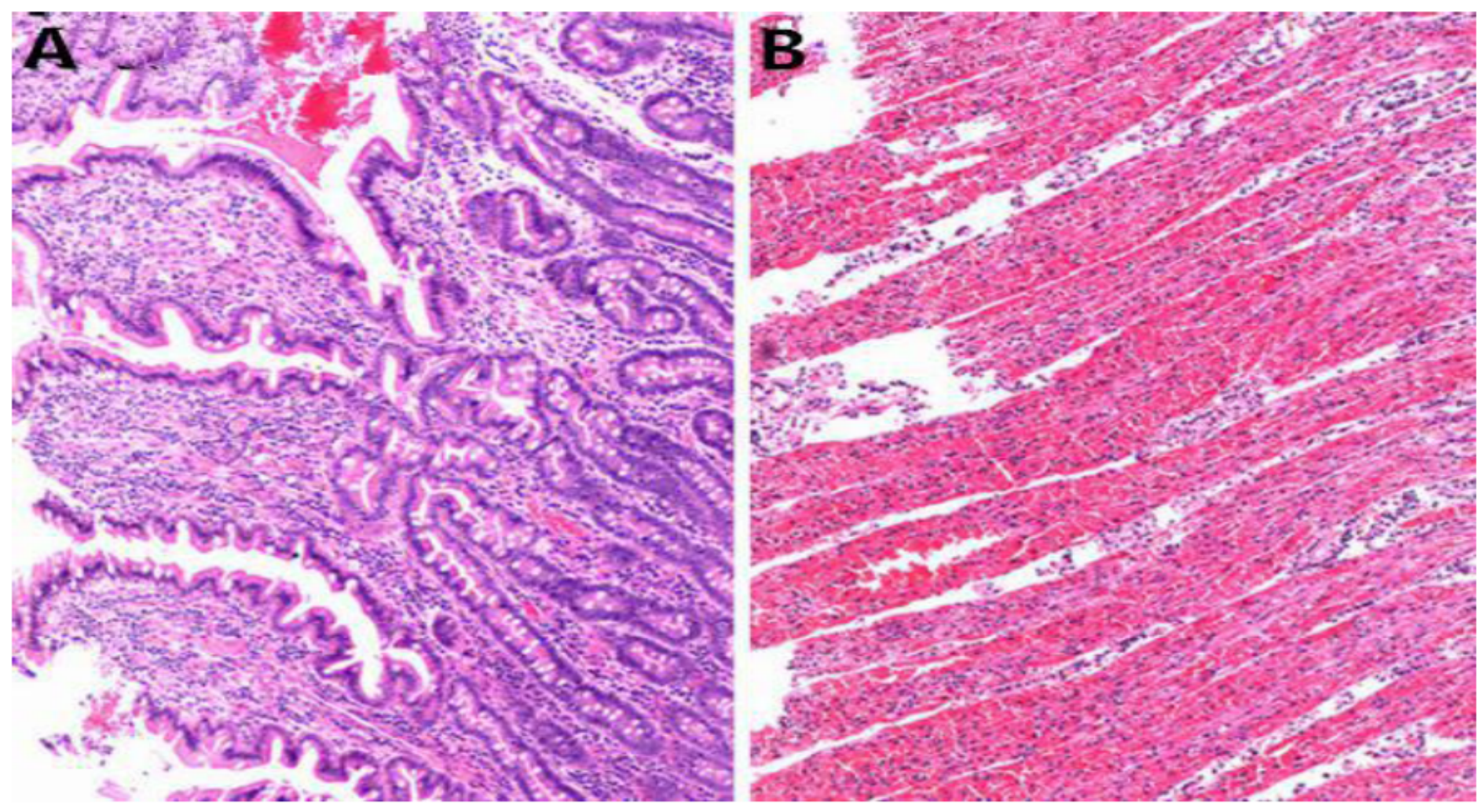


\section{Figure 7}

Pathology of the intestine. A. Intestine without suspected necrosis. B. Intestine with suspected necrosis.

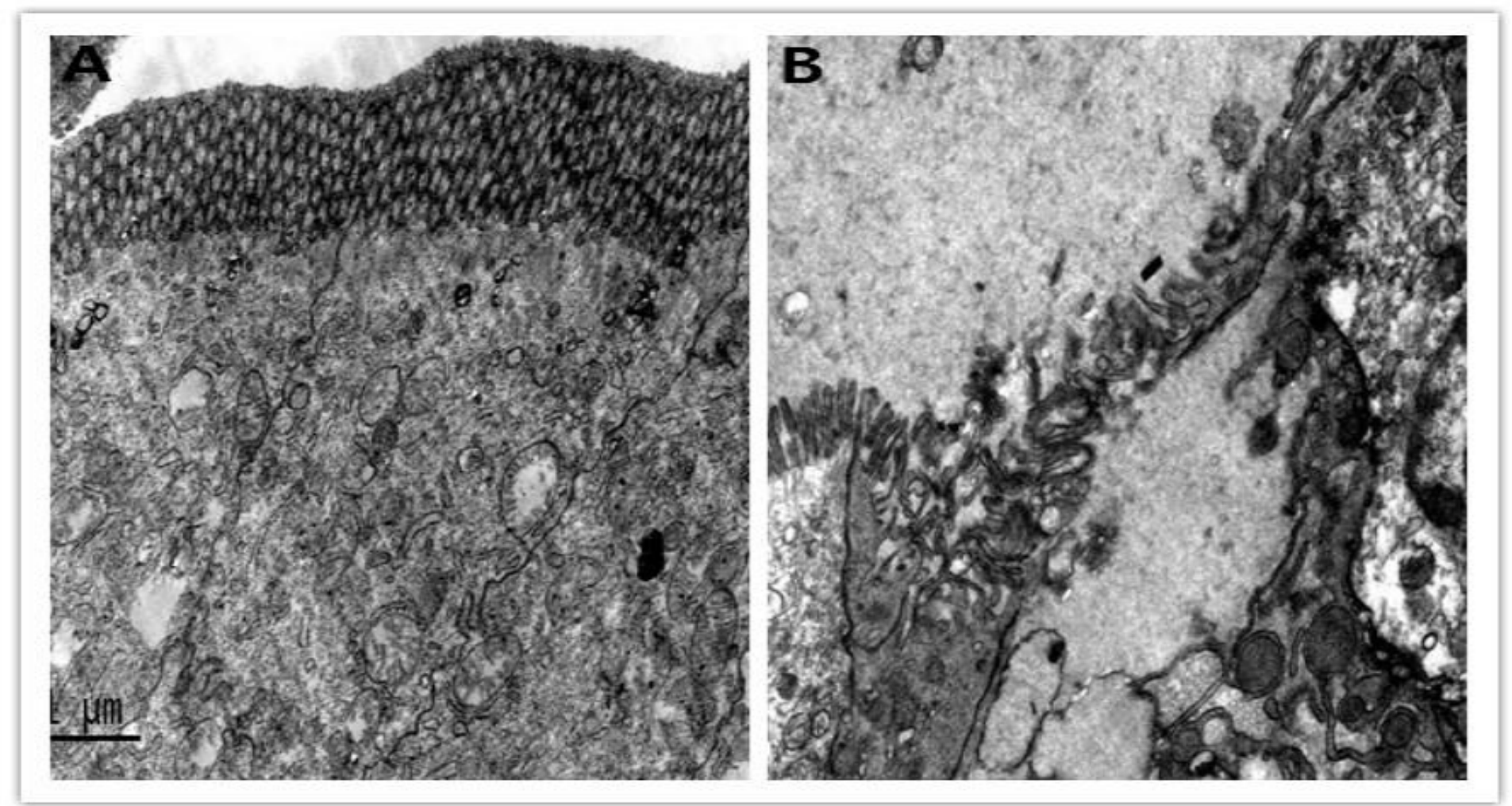

\section{Figure 8}

Transmission electron microscope pathology of the intestine after fixing. A. Position the intestinal tissue at the fixing site before fixation. B. Fix the intestinal tissue at the fixing site 4 hours after emergency surgery. 